\title{
Experience in Diagnostics and Surgical Treatment of Pulmonary Embolism in Pregnant Women
}

\author{
DOI: $10.17691 / \mathrm{stm} 2017.9 .4 .18$
}

Received May 25, 2017

A.P. Medvedev, MD, DSc, Professor, Head of the Department of Hospital Surgery named after B.A. Korolev";

A.L. Maksimov, MD, PhD, Chief Physician²;

S.V. Nemirova, MD, PhD, Associate Professor, Department of Hospital Surgery named after B.A. Korolev'; V.V. Pichugin, MD, DSc, Professor, Head of the Department of Anesthesiology, Resuscitation and Emergency Management';

O.I. Demarin, MD, Cardiovascular Surgeon, Department of Surgical Treatment of Cardiac Rhythm Disorders2;

M.L. Kalinina, MD, PhD, Assistant, Department of Anesthesiology, Resuscitation and Emergency Management ${ }^{\mathrm{T}}$

M.B. Kozina, MD, PhD, Head of the Department of Radiological Diagnostics;

V.A. Chiginev, MD, DSc, Professor, Head of the Department of Acquired Valvular Disease ${ }^{2}$

${ }^{1}$ Nizhny Novgorod State Medical Academy, 10/1 Minin and Pozharsky Square, Nizhny Novgorod, 603005,

Russian Federation;

${ }^{2}$ Specialized Cardiac Surgery Clinical Hospital, 209 Vaneeva St., Nizhny Novgorod, 603136, Russian Federation

Pulmonary embolism (PE) is a serious and frequent extragenital complication of pregnancy, yet too few descriptions of its treatment exist.

This article describes three clinical cases of surgical treatment of pregnant women with the diagnosis of massive critical PE resulted from deep vein thrombosis of the lower extremities and small pelvis with recurrent embolic syndrome. The diagnoses were confirmed with instrumental methods (echoCG, multislice spiral CT angiography). The patients were operated by employing the technique of open thromboembolectomy from the pulmonary arteries on the beating heart.

It was found that results and terms of different therapies are determined by the area of lesion and localization of emboli in pulmonary vascular bed. The optimal surgery technique in this case is embolectomy from pulmonary arteries in conditions of artificial circulation on the beating heart without aortic compression with minimized perfusion time and maintaining high volume velocity $\left(2.4 \mathrm{~L} / \mathrm{min} / \mathrm{m}^{2}\right)$ and average arterial blood pressure $(70-75 \mathrm{~mm} \mathrm{Hg})$ that allows fast and complete recovery of pulmonary hemodynamics. Refusal from the use of cardioplegia contributes to adequate delivery of oxygen to tissues and the fetus; in two cases it was possible to prolong pregnancy with further childbirth, in one case an emergency caesarean section was performed.

The authors note the importance of a thorough examination, a selection of an adequate preoperative therapy and the need for emergency surgery in patients in a severe state. The results of the study show that open embolectomy from pulmonary arteries in case of massive critical PE in pregnant women can save mother's life and ensure the birth of a healthy child.

Key words: pulmonary embolism; PE in pregnant women; embolectomy; thrombolysis.

Pulmonary embolism (PE) is one of the severest extragenital complications of pregnancy. The main causes of maternal mortality in PE are reflex drop in cardiac output, ventricular fibrillation and acute respiratory failure. All pregnant women who are suspected of thromboembolic complications must be hospitalized in specialized clinics. The choice of the therapeutic strategy for pregnant women with PE is one of the most difficult problems in surgical and obstetrical practice. Insufficient verification of the diagnosis can lead to inadequate therapy, whereas X-ray and radiological methods of diagnostics which are a golden standard of screening evaluation are not applicable in early pregnancy and must be used on strict indications. Thrombotic alertness and lack of negligence to "embolic signs" provide a balanced approach to diagnosis verification. The most complicated problems occur in case of massive critical thromboembolism which can be observed in lesion of the trunk and main branches of the pulmonary artery, in PE with a mobile thrombus of the right heart, in the context of cardiogenic shock and the need for resuscitation. Therapeutic measures (thrombolytic therapy and open embolectomy) in these patients must be aimed at fast recovery of perfusion of pulmonary arterial bed with normalization of pressure in the pulmonary artery and the function of the right ventricle, and open embolectomy must be regarded as the last opportunity to save lives of the mother and her child [1-5]. Moreover, patients' management tactics must be worked out by the surgeon and obstetriciangynecologist in cooperation with an anesthesiologist depending on the severity of the patient, localization of

For contacts: Alexandr P. Medvedev, e-mail: medvedev.map@yandex.ru 
the thrombus, gestational age, fetus viability and degree of maturity.

Multiple findings [2-9] testify that often such patients do not get timely medical assistance. The reason for that is complex and includes, in our opinion, lack of knowledge of the latest fundamental changes in the cardiosurgery methods as well as lack of trust or insufficient understanding of possibilities of up-to-date surgical treatment. In the majority of cases (more than $95 \%$ ), for the successful treatment of PE it is enough to administer anticoagulants. As a rule, these are patients with small lesion of pulmonary vascular bed without explicit pulmonary hypertension and dysfunction of the right ventricle. In case of the critical character of embolism with massive pulmonary and cardiac insufficiency, there is a need for the fastest recovery of the blood flow and elimination of dangerous overload of the right heart. It requires fastest possible surgery embolectomy from pulmonary arteries - or thrombolytic therapy. At present, surgical intervention and thrombolytic therapy are considered to be competing methods. Doctors perform either open embolectomy or therapy with activators of fibrinolysis. In our opinion, only objective indications in each particular case can guarantee successful treatment.

Surgical treatment of PE involves complete removal of thromboemboli from the pulmonary vascular bed. Indications for open embolectomy in pregnant women
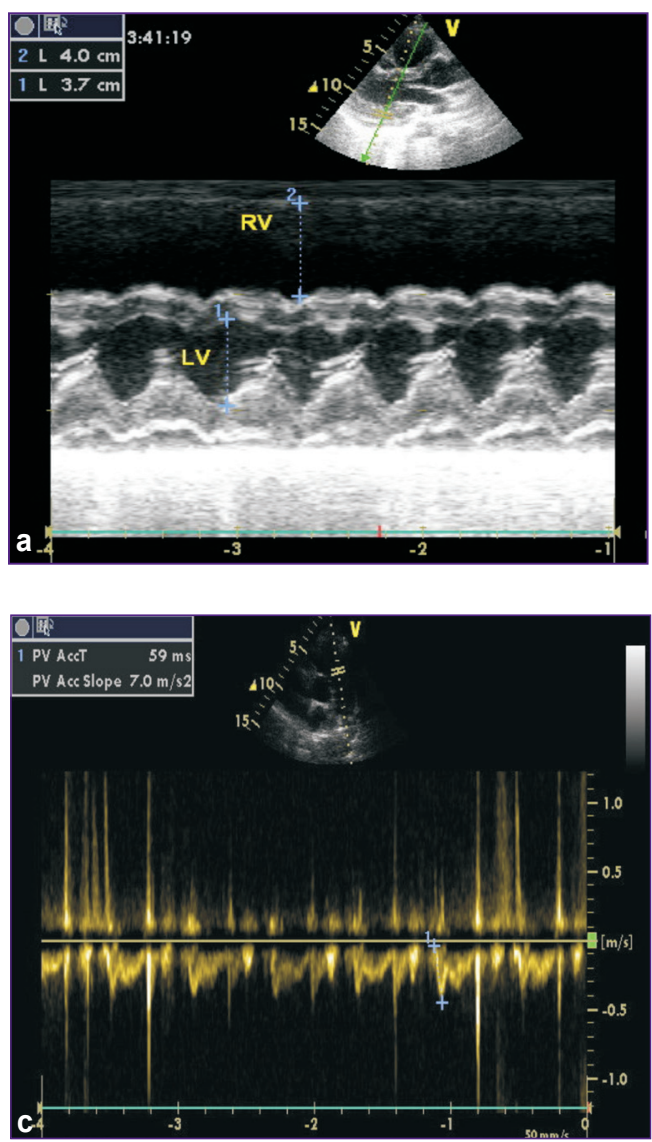

with massive PE do not differ from those for other patients. They are as follows: ineffectiveness of thrombolytic therapy, absolute contraindications to the use of thrombolytics, time shortage for thrombolysis due to a severe state of the patient. A special attention should be paid to the tactics of anesthesiology and perfusion support of emergency operations performed for the treatment of acute massive $\mathrm{PE}$ in pregnant women. The study [10] showed that maternal mortality in pregnant women during operations in conditions of artificial circulation (AC) did not exceed the one in non-pregnant women $(1.47 \%)$ who were operated on during planned surgery. In case of emergent surgery on patients in critical condition with overload of the right heart and unstable hemodynamics, the frequency of maternal mortality increases considerably. However, the main risk of $A C$ is its influence on the fetus.

All the above-mentioned determine the importance of a more critical approach to the treatment of pregnant women with PE. Complexity of the clinical picture, impossibility to collect observation data sufficient for analysis in one institution and, as a result, the lack of clinical recommendations causes serious difficulties in timely selection of indications and surgical tactics. To illustrate surgical treatment specificity of cases of massive critical PE in pregnant women with different clinical courses, we will describe three cases of successful operations on pregnant women to treat

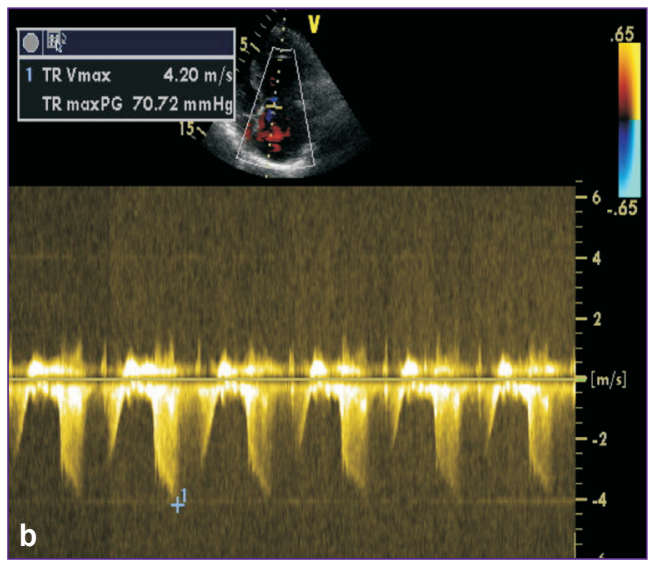

Figure 1. EchoCG of Patient M., 25 years old: (a) M-mode: RV — right ventricle, LV - left ventricle (dilatation of the right ventricle, flattening of interventricular septum, systolic flattening of interventricular septum); (b) continuous-wave Doppler: regurgitation on the tricuspid valve; (c) pulse-wave Doppler: twophase type of blood flow in the output tract of the right ventricle 
acute massive PE (peculiarities of diagnostics, preoperation preparation, anesthesiology-perfusion support of operations, surgical equipment and post-operation follow-up).

The study was performed in accordance with Helsinki Declaration accepted in June 1964 (Helsinki, Finland) and reviewed in October 2000 (Edinburgh, Scotland) and approved by the Ethics Committee of Nizhny Novgorod State Medical Academy. Every patient signed an informed consent form.

\section{Specificity of pulmonary embolism diagnostics in pregnant women}

In all three patients, PE was developed due to thrombosis of deep veins of the lower extremities and small pelvis with recurrent embolic syndrome.

Patient M., 25 years old, $2^{\text {nd }}$ pregnancy. The patient was admitted to the cardiological center by ambulance on November 8, 2013 with the diagnosis "acute massive PE of the main branches of pulmonary arteries; iliofemoral thrombosis on the left; pregnancy 30 weeks". The state of the patient was severe: tachypnea, tachycardia; oxygen therapy via mask, constant infusion of Dopmin. EchoCG on admission revealed evident signs of right ventricle overload: diastolic size (parasternal position) was $40 \mathrm{~mm}$; transverse dimension of the right atria was $46 \mathrm{~mm}$; there was systolic flattening of the interventricular septum, regurgitation degree II on the tricuspid valve. Maximum systolic pressure in the pulmonary artery was $75 \mathrm{~mm} \mathrm{Hg}$, the average pressure was $52 \mathrm{~mm} \mathrm{Hg}$, in the outflow tract of the right ventricle there was registered a two-phase blood flow with explicit incisures and further low-speed phase (Figure 1). There were no thrombotic masses in the right heart and pulmonary trunk.

Multislice spiral CT angiography (Figure 2) visualized extended spindle-shaped defects of filling the main branches of pulmonary arteries that narrow the lumen by $70 \%$, variegated defects of lobar and segmental branches of the right and left branches of pulmonary arteries, dilation of the right heart chambers (signs of overloads of the right heart chambers, indirect signs of pulmonary hypertension).

The patient was consulted by obstetriciangynecologist: the uterus was not excited, fetal heartbeat was clear, heart rate was $145 \mathrm{bpm}$, no signs of fetal hypoxia were registered. There were no obstetric indications for abortion.

A decision was made to perform an emergent operation aimed at recovery of pulmonary hemodynamics.

Patient B., 41 years old, $4^{\text {th }}$ pregnancy. The patient was admitted to a Specialized Cardiosurgical Clinical Hospital of Nizhny Novgorod by ambulance on March 25, 2016 with the diagnosis: "acute massive thromboembolism of the main branches of pulmonary arteries; acute pulmonary heart; iliofemoral thrombosis of the right lower extremity; pregnancy 25 weeks". The patient was in a severe condition with recurrent losses of consciousness, tachypnea and tachycardia at rest. She received divided doses of dexamethasone $(24 \mathrm{mg}$ in total), oxygen therapy was continued via mask. Blood pressure was 110/60 mm Hg, heart rate was $100 \mathrm{bpm}$, oxygen saturation was $96 \%$.

EchoCG revealed signs of the right ventricle overload: diastolic size (parasternal position) was $43 \mathrm{~mm}$; transverse dimension of the right atria was $50 \mathrm{~mm}$; there was systolic flattening of the interventricular septum, regurgitation degree II on the tricuspid valve. Maximum systolic pressure in the pulmonary artery was $65 \mathrm{~mm} \mathrm{Hg}$, the average pressure was $43 \mathrm{~mm} \mathrm{Hg}$, in the outflow tract of the right ventricle there was registered a two-phase blood flow with explicit incisures and further low-speed phase (Figure 3). There were no thrombotic masses in the right heart.

Multislice spiral CT angiography visualizes differentcaliber defects of filling lower lobar branches of the right and left branches of the pulmonary artery, evident congestive changes in the lesser circulation, confluent foci of segment pneumonic infiltrations of the right and left lungs (Figure 4).

The patient was consulted by obstetriciangynecologist: the fetus heartbeat was clear, 140 $\mathrm{bpm}$, the uterus was not excited, no signs of the fetus hypoxia were registered. There were no obstetric contraindications to prolong pregnancy. A decision was made to perform life-saving open embolectomy. The risk of operation was evaluated as extremely high - III-IV according to ASA (American Society of Anesthesiologists) scale.

Patient S., 32 years old, $1^{\text {st }}$ pregnancy. The patient was admitted to hospital on June 15, 2017 because on June 14 acute PE was developed at the background of thrombophlebitis of deep veins of the left lower extremity and 32-week pregnancy. The state at the admission was severe; she had weakness, tachypnea and tachycardia at rest were observed. EchoCG showed the signs of the right ventricle overload, regurgitation degree II on the tricuspid valve with the average calculated pressure in the pulmonary artery being $52 \mathrm{~mm} \mathrm{Hg}$, in the outflow tract of the right ventricle there was registered a twophase blood flow like in the previous cases (Figure 5).

Multislice spiral CT angiography registered defects of filling the branches of the pulmonary artery, the saddleshaped on the right and parietal on the left, narrowing the artery lumen by 55 and $30 \%$, respectively. There were also occlusal defects of filling the lower lobar branches of the pulmonary artery and different-caliber defects of filling segmented branches of the arteries of both lungs, there were indirect signs of pulmonary hypertension (Figure 6).

The patient was consulted by the obstetriciangynecologist, there were no obstetric indications for abortion.

There was made a decision to perform emergent thrombectomy from pulmonary arteries. 

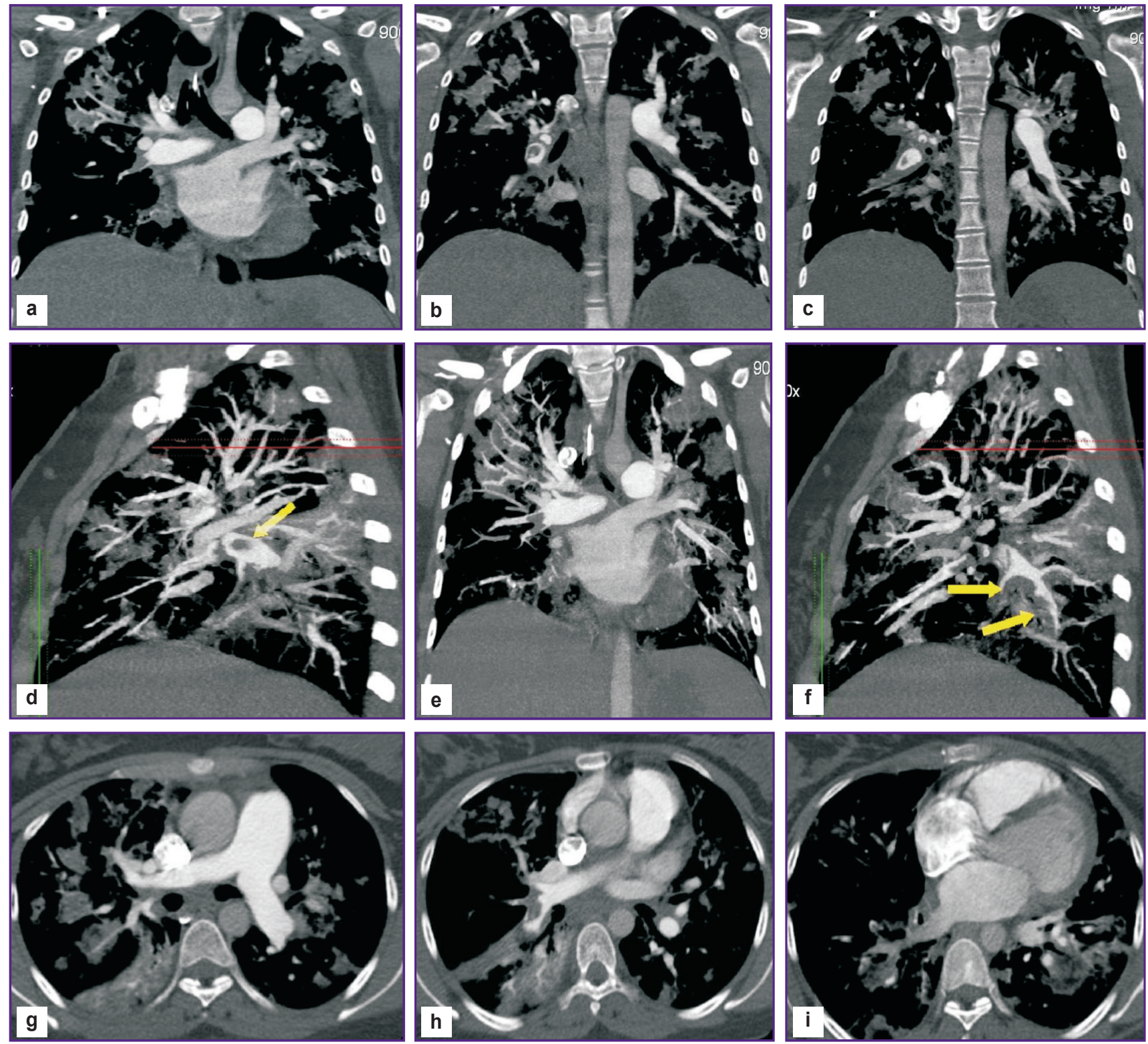

Figure 2. Multislice spiral CT angiography of Patient M., 25 years old:

(a)-(c) MPR reconstructions in the coronal plane; (d)-(f) MIP reconstructions in the coronal and sagittal planes; (g)-(i) MPR reconstruction in the axial plane

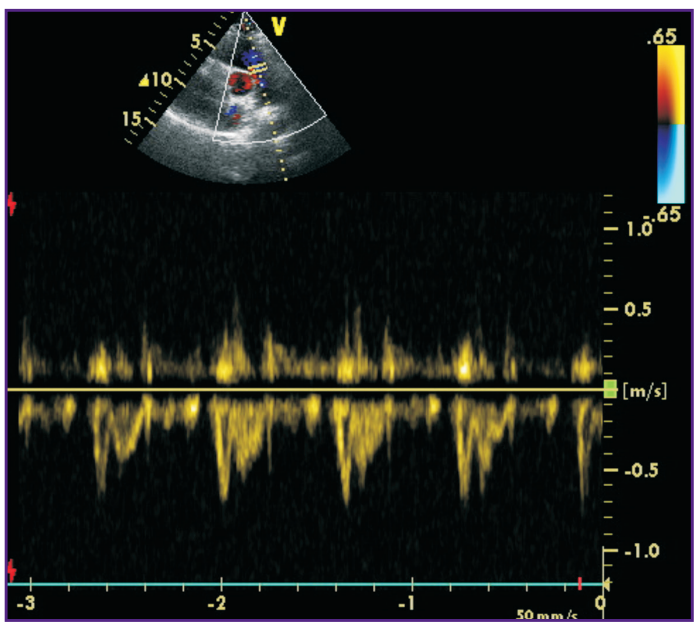

Figure 3. EchoCG of Patient B., 41 years old:

Pulse-wave Doppler: two-phase type of blood flow in the output tract of the right ventricle 

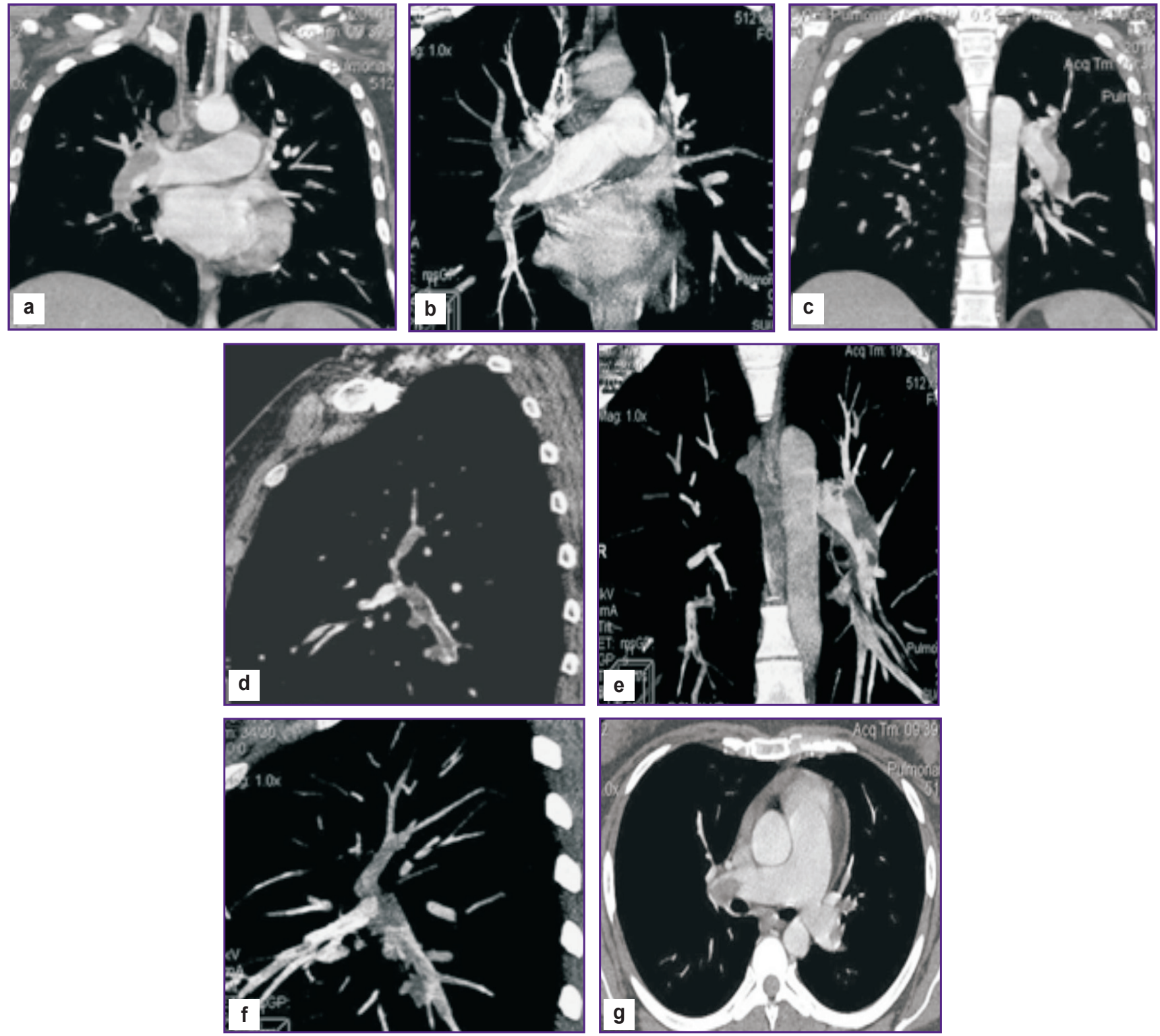

Figure 4. Multislice spiral CT angiography of Patient B., 41 years old:

(a)-(c) MPR reconstructions in the coronal plane; (d)-(f) MIP reconstructions in the coronal and sagittal planes; (g) MPR reconstruction in the axial plane

Figure 5. EchoCG of Patient S., 32 years old:

Pulse-wave Doppler: two-phase type of blood flow in the output tract of the right ventricle

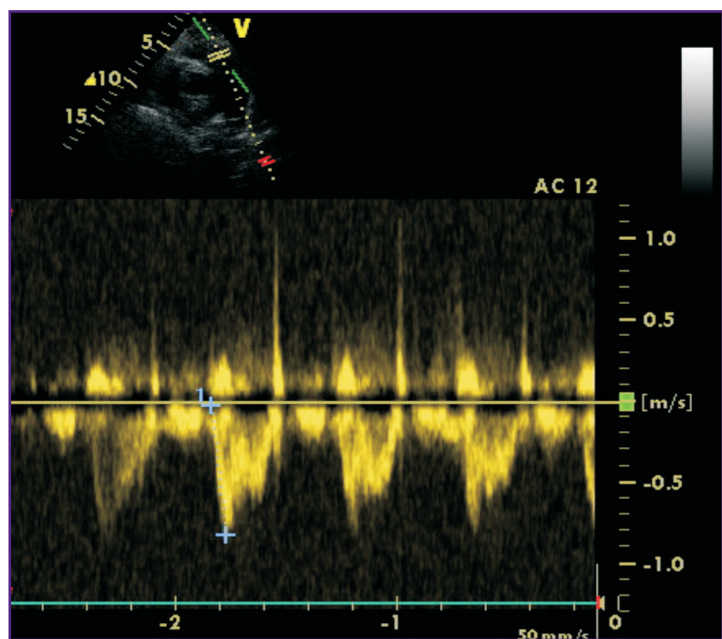



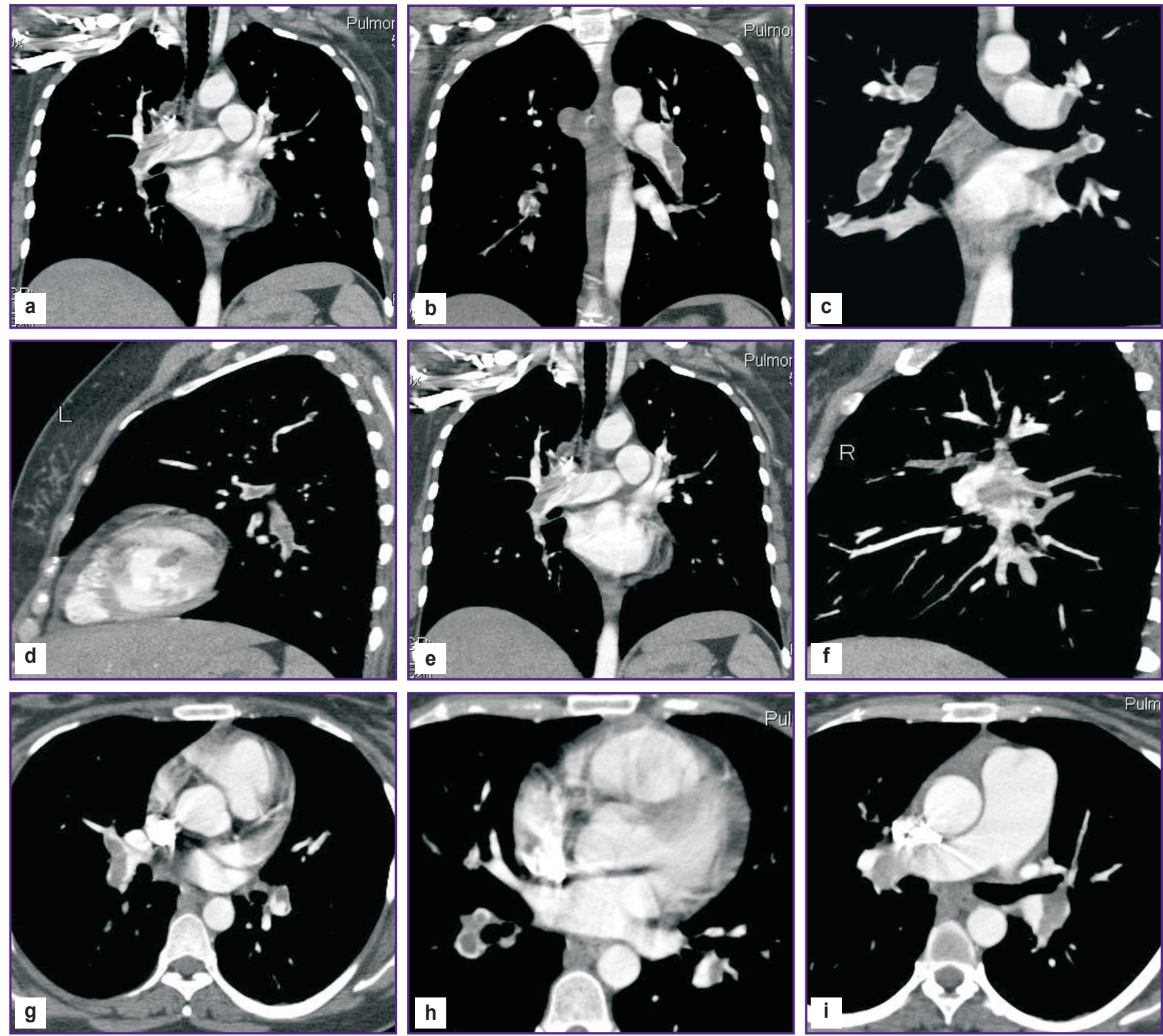

Figure 6. Multislice spiral CT angiography of Patient S., 32 years old:

(a)-(c) MPR reconstructions in the coronal plane; (d)-(f) MIP reconstructions in the coronal and sagittal planes; (g)-(i) MPR reconstruction in the axial plane

\section{Peculiarities of pre-operational preparation and anesthesiology-perfusion support of the operation}

Pre-operational preparation of pregnant women included evaluation of the function of cardiovascular system and its necessary optimization; correction of metabolism disorders (water-electrolyte balance, acidbase state, blood gases); evaluation and optimization of the utero-placental blood flow (obstetriciangynecologist); prescription of glucocorticosteroids (dexamethasone 4-8 $\mathrm{mg} /$ day). The obstetriciangynecologist was necessarily involved in evaluation of the state of the pregnant women and their fetuses. In all cases before the operation pregnant women had a viable fetus.

Two patients were admitted directly to the operating room, one was admitted to the Department of Resuscitation and Intensive Therapy. The women's state before the operation was assessed as extremely severe - class IV according to ASA classification.

Monitoring of the mothers' state included first and foremost control of the state of the cardiovascular and respiratory systems: a 6-lead ECG, invasive measurement of blood pressure and central venous pressure; pressure in the pulmonary artery, blood saturation with hemoglobin, $\mathrm{CO}_{2}$ concentration, respiratory volume and pulmonary minute volume, 
pressure in the airways, minimal alveolar concentration, compliance of the lungs were performed according to indications. The following parameters were investigated in all the patients during the operations and during the immediate post-operation period: acid-base state, blood gases, hemoglobin, hematocrit, activated clotting time, coagulogram, electrolytes, total protein, lactate. In the post-operation period markers of myocardium damage (CK $\mathrm{MB}, \mathrm{CK}$ ) were additionally evaluated as well as the function of kidneys and liver and oxygen transport function of blood. Monitoring of the fetal state included cardiotocography that allowed monitoring of the fetal heart rate and measurement of the contractile activity of the uterus with TOCO-sensor.

Premedication was not performed due to emergency situation and unstable hemodynamics.

For induction into anesthesia in all cases the combination of Diazepam $(0.10 \pm 0.01 \mathrm{mg} / \mathrm{kg})$ and Propofol $(2.0 \pm 0.4 \mathrm{mg} / \mathrm{kg})$ was used. The trachea was intubated at the background of muscle relaxation with pancuronium bromide $(0.1-0.12 \mathrm{mg} / \mathrm{kg})$. After the induction, to prevent uterine displacement the patient was placed lying on the side with a left turn of $15^{\circ}$ with a wedge under the right thigh or lying on the table with the left side tilt. Anesthesia before AC, during perfusion and after the perfusion was maintained with sevoflurane (1-3 vol.\%), at the traumatic stages of the operation 50$100 \mu \mathrm{g}$ of fentanyl was administered in divided doses, its total dosage did not exceed $10 \mu \mathrm{g}$. The neuromuscular block was maintained by fractional infusion of pancuronium bromide of $2 \mathrm{mg}$ each till the total dosage reached $0.2 \mathrm{mg} / \mathrm{kg}$.

Changes in the central hemodynamics at the induction into anesthesia were irrelevant and were accompanied by relative stability of values of blood pressure, central venous pressure, cardiac output. During the further course of pre-perfusion period there was registered a relevant drop in the average $\mathrm{BP}$, heart rate growth, decrease in cardiac and percussion indexes of the heart. However, we associate these changes not only with cardiodepressant action of the anesthetics used, but also with a serious disorder of intracardiac hemodynamics caused by the main disease at the background of surgical manipulations.

Before the beginning of $A C$, heparinization in the dose of $2-3 \mathrm{mg} / \mathrm{kg}$ of the body weight was performed. AC was done on the device Stockert (Sorin Group Deutschland, Germany) with membrane oxygenators Medtronic (Medtronic Inc., USA). In all cases non-pulsating flow in the normothermia mode was used. All perfusions were performed at an increased (up to $2.8 \mathrm{~L} / \mathrm{min} / \mathrm{m}^{2}$ ) volume velocity, which allowed us to maintain an average BP higher than $80 \mathrm{~mm} \mathrm{Hg}$. Ultrahemofiltration was used in all operations.

During normothermic perfusions, an increase in oxygen concentration by $10-15 \%$, both in arterial and venous blood was registered, while arteriovenous oxygen difference decreased and oxygen transport increased; there were insignificant changes in the tissue extraction factor. Undoubtedly, this testifies a more favorable oxygen perfusion mode both for the mother and her fetus.

In post-perfusion period, to stabilize hemodynamics and in the cases of acute heart failure (in two patients) we used $\beta$-adrenomimetic drugs (Dopmin in the dose of $3-5 \mu \mathrm{g} / \mathrm{kg} / \mathrm{min}$ ) in combination with nitrates and glucocorticoids (Dexasone 12-20 mg). Correction of the blood coagulation system was done with protaminesulfate under the control of activated clotting time. Ultrahemofiltration performed during AC allowed controlling the degree of hemodilution: at the end of $\mathrm{AC}$, hemoglobin was $105.0 \pm 8.1 \mathrm{~g} / \mathrm{L}$, and hematocrit was $28.7 \pm 5.6 \%$. Furthermore, we took measures to lower intraoperational blood loss; total blood loss did not exceed $576.0 \pm 65.0 \mathrm{ml}$.

In the post-operation period, all patients underwent prolonged artificial lung ventilation; its duration was from 10 to $48 \mathrm{~h}$. A special attention was paid to adequate correction of post-operational disorders of myocardium contractibility and maintenance of the adequate level of blood oxygen-transport function. We did not use a-agonists (Noradrenalin, Mesaton) for inotropic stimulations, but we used a combinations of $\beta$-agonists (dopamin, dobutamin) with vasodilators (nitroglycerin, isosorbide dinitrate).

The patients' follow-up after the operation involved consultations by obstetrician-gynecologist. From the first days we arranged measures to prevent termination of their pregnancies. Thus, we prescribed antispasmodics, sulfurous magnesia and non-steroid anti-inflammatory drugs (indomethacin), tocolytic Ginipral, vitamin E.

\section{Complications of the post-operation period}

All the patients underwent open embolectomy from the main and lobar pulmonary arteries under the conditions of normothermia on a beating heart without aorta compression. In the first case (Patient M., 25 years old), it was a life-saving operation performed three hours after the admission and involving removal of large emboli, which were heterogeneous in terms of formation time (Figure 7).

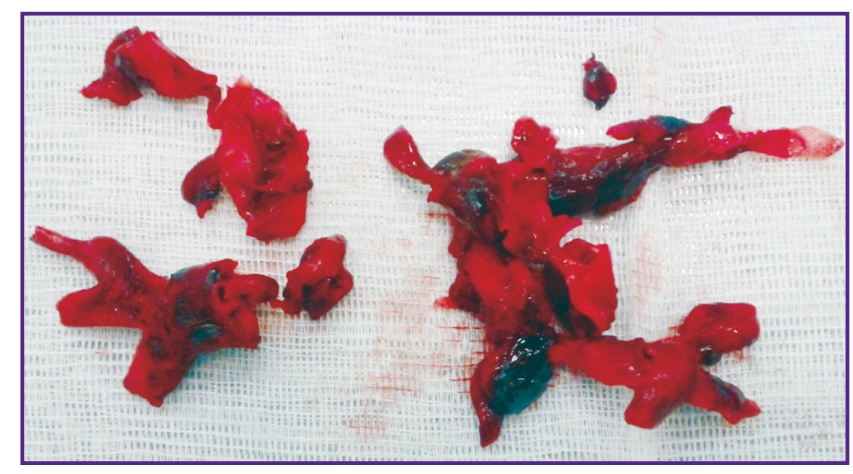

Figure 7. Emboli removed from the main and lobar pulmonary arteries of Patient M., 25 years old 


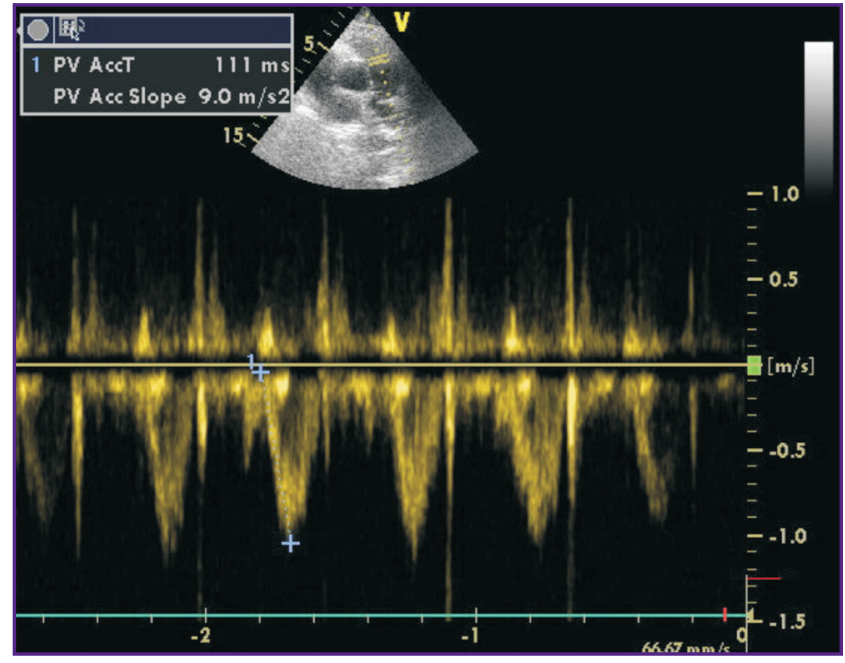

Figure 8. EchoCG of of Patient M., 25 years old:

Pulse-wave Doppler: blood flow in the output tract of the right ventricle in the post-operation period

Post-operation period was smooth, on the next day after the operation the following parameters were achieved: normalization of blood flow dopplergrams in the output tract of the right ventricle in the form of an isosceles triangle, lowered degree of regurgitation on the tricuspid valve up to degree I, decrease in the average pressure in the pulmonary artery up to $29 \mathrm{~mm} \mathrm{Hg}$ (Figure 8). The cavity of the right ventricle $(37 \mathrm{~mm})$ and the right atrium $(43 \mathrm{~mm})$ remained increased.

On day 12 after the operation, the patient was discharged for outpatient treatment in a satisfactory state. One month after the operation, the sizes of the cavities of the right ventricle and the right atrium were normalized.

In week 37 of pregnancy the patient underwent caesarean section according to obstetric indications (uterine scar) and gave birth to a healthy baby girl.

In the second case (Patient B., 41 years old), was operated on $2 \mathrm{~h}$ after the admission, the postoperation period was also smooth. The next day after the operation, the following parameters were achieved: normalization of blood flow dopplergrams in the output tract of the right ventricle in the form of an isosceles triangle, lowered degree of regurgitation on the tricuspid valve up to degree I, decrease in the average pressure in the pulmonary artery up to $34 \mathrm{~mm} \mathrm{Hg}$. The cavity of the right ventricle $(41 \mathrm{~mm})$ and the right atrium $(47 \mathrm{~mm})$ remained increased.

On day 12, the woman was discharged for outpatient treatment. One month after the operation, normalization of all the echoCG parameters (Figure 9) was observed. In week 39, the woman delivered a healthy baby girl by vaginal birth.

In the third case (Patient S., 32 years old), was also operated on right after the admission and identification of emboli localization in the pulmonary arteries. The

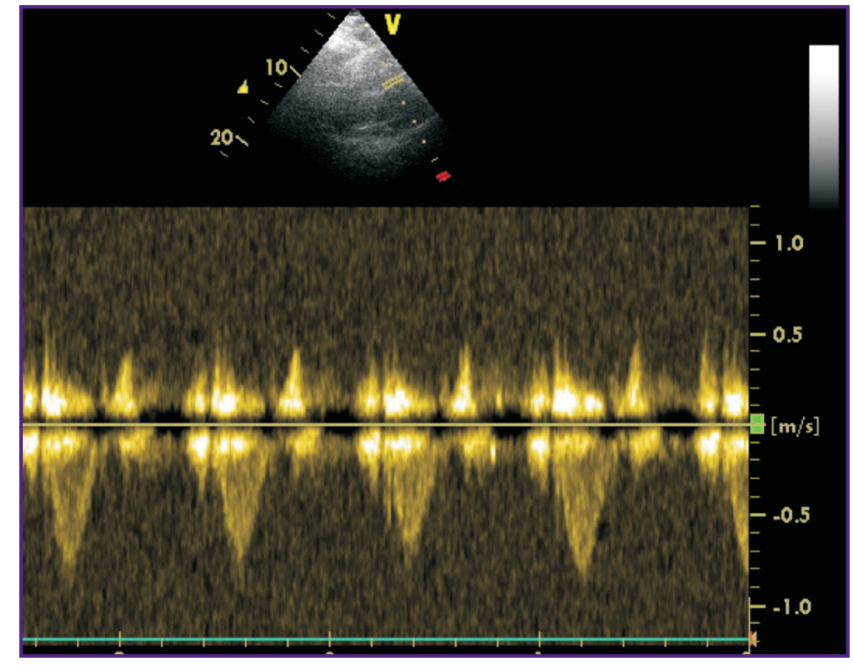

Figure 9. EchoCG of Patient B., 41 years old:

Pulse-wave Doppler: blood flow in the output tract of the right ventricle in the post-operation period

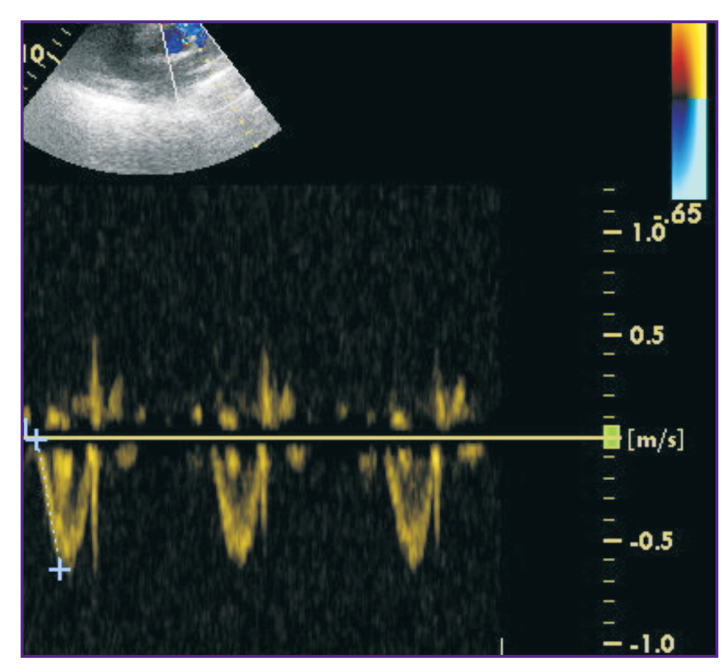

Figure 10. EchoCG of Patient S., 32 years old:

Pulse-wave Doppler: blood flow in the output tract of the right ventricle in the post-operation period

operation resulted in restoration of a good retrograde blood flow, though in the early post-operation period CT revealed right-sided lower lobar infarct-pneumonia which resulted in an emergency caesarean section. Postoperation period was without complications, the patient and her baby boy were discharged in a satisfactory state. In the longer post-operation period, there was registered a change in the form of blood flow in the output tract of the right ventricle from two-phase to one-phase as well as the decreased average pressure to $34 \mathrm{~mm} \mathrm{Hg}$ (Figure 10).

\section{Discussion}

The complexity of treating pregnant women with $\mathrm{PE}$ is determined by a special medical and social status 
of these women and serious restrictions that make the doctor exclude and minimize the negative impact of diagnostic and therapeutic manipulations on the fetus. The provided examples show different variants of clinical development of massive critical PE with evident lifethreatening hemodynamic and respiratory disorders. These cases had some common features, such as thrombosis of deep veins preceding PE and the need for basic anticoagulant therapy with low-molecular heparin. These examples also showed that it is a mistake to think that it is enough to use only anticoagulant therapy without checking the character of the thrombus and risks of its transfer to embolus-dangerous forms for patients with thrombotic processes in the inferior vena cava system.

In the majority of cases, an instrumental examination of the pulmonary vascular bed is required to confirm the diagnosis of PE and to evaluate the area of embolic lesion.

Therefore, it is important to have a preliminary clinical evaluation and ultrasound examination and determine the character of blood flow in the pulmonary artery and calculate the pressure in it. It can help doctors justify the need for multislice spiral $\mathrm{CT}$, which has a radiation effect on the developing fetus in early terms, because the consequences of undiagnosed PE can be fatal [11].

The classical symptoms described in many manuals and textbooks are shortness of breath, chest pain, hemoptysis, syncopic conditions, arterial hypotension, cyanosis of the face and upper half of the body. However, the combination of "classical" signs in the clinical picture of thromboembolism can be a "happy" case for diagnostics, as it occurs in less than $10 \%$ of patients [1]. A probability of PE is increased if there is thrombosis of deep veins and evident hypertension of the small circulation verified with ultrasound investigations. The area of lesion and localization of thromboemboli in the pulmonary vascular bed as well as explicitness of acute pulmonary hypertension define indications and terms of different therapeutic measures. These parameters in pregnant women should be investigated extremely thoroughly.

Anticoagulant therapy is the basis of the conservative treatment of PE. As anticoagulant drugs for pregnant women one uses unfractionated low-molecular heparins that do not penetrate the placenta and do not have a negative impact on the fetus. In case of massive critical PE (the lesion area of the pulmonary vascular bed is more than $70 \%$ ) with the development of severe cardiopulmonary insufficiency and high risk of death one considers possibilities of thrombolytic therapy, selective intra-arterial thrombolysis with destruction and aspiration of thromboemboli as well as open embolectomy. The publications analyzing these variants of treatment provide mainly a series of descriptions of clinical observations [12-14].

Due to a high risk of bleeding, thrombolytic therapy is not a routine method of treating massive PE. The study
[15] describes 13 cases of using thrombolytic therapy in pregnant women. Among the patients, there were no cases of the mothers' deaths, but there were registered 4 bleedings, 2 fetal deaths and 5 pre-term deliveries. It is considered that such frequency of complications is much lower than the risk of death in case of massive critical PE without thrombolysis. It serves as a kind of "justification" for the clinician who chose this tactics [1].

The literature also mentions successful attempts of selective thrombolysis with destruction and aspiration of thromboemboli in massive PE during pregnancy [16-18]. In these reports, the authors stress the advantages of this tactics, i.e. reduced systemic effects, predictable removal of thromboemboli and lower risks for women and the developing fetuses. However, there is no absolute confidence in the fact that catheter thrombolytic therapy is more preferable than systemic thrombolysis and heparin-therapy. Transcatheter thrombectomy allows fragmenting embolic material which can further have a high probability of getting into the periphery pulmonary system and increasing the risk of pulmonary hypertension and its chronization [19].

Surgical treatment of PE involves a complete removal of thromboemboli from the pulmonary vascular bed resulting in normalization of pressure in the pulmonary artery and the function of the right ventricle.

Thus, on the basis of the acquired findings it is difficult to draw, a conclusion about the optimal method of treating massive critical PE in pregnant women. The authors accumulated wide clinical experience of treating PE, which includes 150 performed surgeries on the patients with acute and recurrent obstruction of pulmonary arteries and high hypertension of small circulation. The analysis of our data (lack of lethality for the last six years in more than 120 embolectomies), as well as the experience of Russian and foreign colleagues, allows us to consider open embolectomy the most appropriate method of treating pregnant women, because they give an opportunity not only to save a woman's life, but also to prolong pregnancy and deliver a healthy child. The optimal variant of surgery is embolectomy from pulmonary arteries in conditions of $\mathrm{AC}$ on the beating heart without the aorta compression [19-21]. Beyond any doubts it requires an operation under the conditions of a specialized cardio-surgical hospital by surgeons experienced in operations on the heart.

The position of a pregnant woman on the operation table is of great importance [22]. Therefore, to prevent uterine displacement and the development of aortocaval compression, it is essential to place the patient lying on the side with a left turn of $15^{\circ}$ with a wedge under the right thigh or lying on the table with the left side tilt. Such position on the operation table is a requirement for every pregnant woman with gestation term more than 20 weeks to exclude deterioration of uteroplacental blood flow [23]. For all operations we used this position, and it prevented compression of the main vessels by the 
pregnant uterus and maintained uteroplacental blood flow.

The changes in the organ functions caused by pregnancy lead to the change in maternal pharmacodynamics and pharmacokinetics of the drugs [24]. Hemodilution at the beginning of AC further changes pharmacology of the drug and results in an increased variability of unconnected concentrations of the drug. Penetration of the drug via placenta into the fetus occurs according to the gradient of drug concentration from the mother into the fetal circulation and peculiarities of placental membrane functioning. The drugs require more frequent administration without changes in daily dosages. All inhalation anesthetics and the majority of intravenous anesthetizing drugs are fat-soluble and freely penetrate through placenta. Inhalation anesthetics are also strong uterine relaxants and can reduce uterine blood flow. In our studies we did not note the need for considerable increase in the doses of the drugs used for anesthesia. However, it is considered that the most unfavorable maternal and fetal outcomes in operations on the heart during pregnancy are connected with $\mathrm{AC}$ and the state of the mother's cardiovascular system, but not with the drug for anesthesia and its dosage [22].

According to the studies, perfusion with high volume velocity, high perfusion pressure, in a normothermic mode and, if possible, a short one, is the best perfusion strategy to ensure an adequate placental homeostasis [22]. The perfusion tactics we used complies with this strategy completely: normothermic AC, minimization of perfusion time, maintenance of high volume velocity $2.4 \mathrm{~L} / \mathrm{min} / \mathrm{m}^{2}$ and the average arterial pressure $70-75 \mathrm{~mm} \mathrm{Hg}$. Furthermore, refusal from the use of cardioplegia and operating on a beating heart allowed us not only to maintain an optimal level of hemoglobin and hematocrit, but also to provide adequate delivery of oxygen to the tissues and fetus.

In the period from 1970 to 2014 , we found 13 cases of embolectomy in pregnant women described in the available literature. All the patients had massive PE with the growing pulmonary heart insufficiency which was an indication for an operation [25]. Among those operated patients, 2 patients died [26, 27], in 3 cases the fetus died [27-29]. Nonetheless, we agree with Cooley et al. [30] and Hajj-Chahine et al. [31], in that only timely adequate embolectomy from the pulmonary artery, which is possible in case of early PE diagnostics, can save the mother's life and prolong her pregnancy.

Conclusion. Our own experience of three successful operations on pregnant women allows us to make the following conclusions. Surgical treatment of pulmonary embolism in these categories of patients must include:

an early diagnostic examination of the patients suspected of pulmonary embolism, if possible, and including ultrasound investigation evaluating the level of hemodynamic disorders and multislice spiral CT with exact verification of the emboli location in the pulmonary vascular bed; an operation on the beating heart in conditions of normothermic artificial circulation, refusal from cardioplegia, minimization of perfusion time, maintaining its high volume velocity $2.4 \mathrm{~L} / \mathrm{min} / \mathrm{m}^{2}$ and the average arterial blood pressure $70-75 \mathrm{~mm} \mathrm{Hg}$;

maximum possible disobliteration of pulmonary arteries with minimal trauma and duration of intervention on pulmonary vessels.

only after a favorable course of post-operation period with normalization of pressure in the pulmonary artery it is possible to prolong pregnancy.

Financing of the Study and Conflicts of Interest. The study was not financed by any sources; the authors declare no conflict of interest.

\section{References}

1. Tromboemboliya legochnykh arteriy: kak lechit $i$ predotvrashchat' [Pulmonary embolism: how to treat and prevent it]. Pod red. Kirienko A.I., Chernyavskogo A.M., Andriyashkina V.V. [Kirienko A.I., Chernyavskiy A.M., Andriyashkin V.V. (editors)]. Moscow: MIA; 2015; 280 p.

2. Fukuda I., Taniguchi S., Fukui K., Minakawa M., Daitoku K., Suzuki Y. Improved outcome of surgical pulmonary embolectomy by aggressive intervention for critically ill patients. Ann Thorac Surg 2011; 91(3): 728-732, https://doi. org/10.1016/j.athoracsur.2010.10.086.

3. Sareyyupoglu B., Greason K.L., Suri R.M., Keegan M.T., Dearani J.A., Sundt T.M. A more aggressive approach to emergency embolectomy for acute pulmonary embolism. Mayo Clin Proc 2010; 85(9): 785-790, https://doi. org/10.4065/mcp.2010.0250.

4. Kadner A., Schmidli J., Schönhoff F., Krähenbühl E., Immer F., Carrel T., Eckstein F. Excellent outcome after surgical treatment of massive pulmonary embolism in critically ill patients. J Thorac Cardiovasc Surg 2008; 136(2): 448-451, https://doi.org/10.1016/j.jtcvs.2007.11.021.

5. Amirghofran A.A., Emami Nia A., Javan R. Surgical embolectomy in acute massive pulmonary embolism. Asian Cardiovasc Thorac Ann 2007; 15(2): 149-153, https://doi. org/10.1177/021849230701500214.

6. Leacche M., Unic D., Goldhaber S.Z., Rawn J.D., Aranki S.F., Couper G.S., Mihaljevic T., Rizzo R.J., Cohn L.H., Aklog L., Byrne J.G. Modern surgical treatment of massive pulmonary embolism: results in 47 consecutive patients after rapid diagnosis and aggressive surgical approach. $J$ Thorac Cardiovasc Surg 2005; 129(5): 1018-1023, https://doi. org/10.1016/j.jtcvs.2004.10.023.

7. Vohra H.A., Whistance R.N., Mattam K., Kaarne M., Haw M.P., Barlow C.W., Tsang G.M., Livesey S.A., Ohri S.K. Early and late clinical outcomes of pulmonary embolectomy for acute massive pulmonary embolism. Ann Thorac Surg 2010; 90(6): 1747-1752, https://doi.org/10.1016/j. athoracsur.2010.08.002.

8. Konstantinov I.E., Saxena P., Koniuszko M.D., Alvarez J., Newman M.A. Acute massive pulmonary embolism with cardiopulmonary resuscitation: management and results. Tex Heart Inst J 2007; 34(1): 41-46.

9. Goldhaber S.Z. Surgical pulmonary embolectomy: the resurrection of an almost discarded operation. Tex Heart Inst $J$ 2013; 40(1): 5-8.

10. Martin S.R., Foley M.R. Intensive care in obstetrics: an 
evidence-based review. Am J Obstet Gynecol 2006; 195(3): 673-689, https://doi.org/10.1016/j.ajog.2006.05.042.

11. Kucher N., Rossi E., De Rosa M., Goldhaber S.Z. Massive pulmonary embolism. Circulation 2006; 113(4): 577582, https://doi.org/10.1161/circulationaha.105.592592.

12. Sato T., Kobatake R., Yoshioka R., Fuke S., Ikeda T., Saito H., Maekawa K., Hioka T. Massive pulmonary thromboembolism in pregnancy rescued using transcatheter thrombectomy. Int Heart J 2007; 48(2): 269-276, https://doi. org/10.1536/ihj.48.269.

13. Torbicki A., Perrier A., Konstantinides S., Agnelli G., Galiè N., Pruszczyk P., Bengel F., Brady A.J., Ferreira D., Janssens U., Klepetko W., Mayer E., Remy-Jardin M., Bassand J.P.; ESC Committee for Practice Guidelines (CPG). Guidelines on the diagnosis and management of acute pulmonary embolism: the Task Force for the Diagnosis and Management of Acute Pulmonary Embolism of the European Society of Cardiology (ESC). Eur Heart J 2008; 29(18): 22762315, https://doi.org/10.1093/eurheartj/ehn310.

14. Ahearn G.S., Hadjiliadis D., Govert J.A., Tapson V.F. Massive pulmonary embolism during pregnancy successfully treated with recombinant tissue plasminogen activator: a case report and review of treatment options. Arch Intern Med 2002; 162(11): 1221, https://doi.org/10.1001/archinte.162.11.1221.

15. Te Raa G.D., Ribbert L.S.M., Snijder R.J., Biesma D.H. Treatment options in massive pulmonary embolism during pregnancy; a case-report and review of literature. Thromb Res 2009; 124(1): 1-5, https://doi.org/10.1016/j. thromres.2009.03.001.

16. Weinberg L., Kay C., Liskaser F., Jones D., Tay S., Jaffe S., Seevanayagam S., Doolan L. Successful treatment of peripartum massive pulmonary embolism with extracorporeal membrane oxygenation and catheter-directed pulmonary thrombolytic therapy. Anaesth Intensive Care 2011; 39(3): 486-491.

17. O'Keeffe S.A., McGrath A., Ryan J.M., Byrne B. Management of a massive pulmonary embolism in a pregnant patient with mechanical fragmentation followed by delayed catheter-directed thrombolysis in the early postpartum period. J Matern Fetal Neonatal Med 2008; 21(8): 591-594, http:// dx.doi.org/10.1080/14767050802165604.

18. Bechtel J.J., Mountford M.C., Ellinwood W.E. Massive pulmonary embolism in pregnancy treated with catheter fragmentation and local thrombolysis. Obstet Gynecol 2005; 106(5 Pt 2): 1158-1160.

19. Dauphine C., Omari B. Pulmonary embolectomy for acute massive pulmonary embolism. Ann Thorac Surg 2005; 79(4): 1240-1244, https://doi.org/10.1016/j.athoracsur.2004.08.081.

20. Nemirova S., Medvedev A., Pichugin V., Bhandari K.
Surgical embolectomy in massive acute pulmonary embolism: our experience. Nepalese Heart Journal 2015; 12(2): 83, https://doi.org/10.3126/njh.v12i2.13387.

21. Taniguchi S., Fukuda I., Minakawa M., Watanabe K., Daitoku K., Suzuki Y. Emergency pulmonary embolectomy during the second trimester of pregnancy: report of a case. Surg Today 2007; 38(1): 59-61, https://doi.org/10.1007/ s00595-007-3570-0.

22. Chandrasekhar S., Cook C.R., Collard C.D. Cardiac surgery in the parturient. Anesth Analg 2009; 108(3): 777-785, https://doi.org/10.1213/ane.0b013e31819367aa.

23. John A.S., Gurley F., Schaff H.V., Warnes C.A., Phillips S.D., Arendt K.W., Abel M.D., Rose C.H., Connolly H.M. Cardiopulmonary bypass during pregnancy. Ann Thorac Surg 2011; 91(4): 1191-1196, https://doi.org/10.1016/j. athoracsur.2010.11.037.

24. Qasqas S.A., McPherson C., Frishman W.H., Elkayam U. Cardiovascular pharmacotherapeutic considerations during pregnancy and lactation. Cardiol Rev 2004; 12(5): 240-261, https://doi.org/10.1097/01.crd.0000102421.89332.43.

25. Saeed G., Möller M., Neuzner J., Gradaus R., Stein W., Langebrake U., Dimpfl T., Matin M., Peivandi A. Emergent surgical pulmonary embolectomy in a pregnant woman: case report and literature review. Tex Heart Inst J 2014; 41(2): 188194, https://doi.org/10.14503/thij-12-2692.

26. Girz B.A., Heiselman D.E. Fatal intrapartum pulmonary embolus during tocolysis. Am J Obstet Gynecol 1988; 158(1): 145-146, https://doi.org/10.1016/0002-9378(88)90798-3.

27. Lau G. A case of sudden maternal death associated with resuscitative liver injury. Forensic Sci Int 1994; 67(2): 127132, https://doi.org/10.1016/0379-0738(94)90327-1.

28. Marcinkevicius A., Sirvydis V., Triponis V., Baublys A., Martinkenas G., Matulionis A. Pulmonary embolectomy during pregnancy. J Cardiovasc Surg (Torino) 1970; 11(5): 355-338.

29. Duff P., Greene V.P. Pregnancy complicated by solid-papillary epithelial tumor of the pancreas, pulmonary embolism, and pulmonary embolectomy. Am J Obstet Gynecol 1985; 152(1): 80-81, https://doi.org/10.1016/s00029378(85)80182-4

30. Cooley D.A., Bell A.C. Jr., Alexander J.K. Acute massive pulmonary embolism. Successful surgical treatment using temporary cardiopulmonary bypass. JAMA 1961; 177(5): 283286, https://doi.org/10.1001/jama.1961.03040310001001.

31. Hajj-Chahine J., Jayle C., Tomasi J., Corbi P. Successful surgical management of massive pulmonary embolism during the second trimester in a parturient with heparin-induced thrombocytopenia. Interact Cardiovase Thorac Surg 2010; 11(5): 679-681, https://doi.org/10.1510/ievts.2010.247460. 